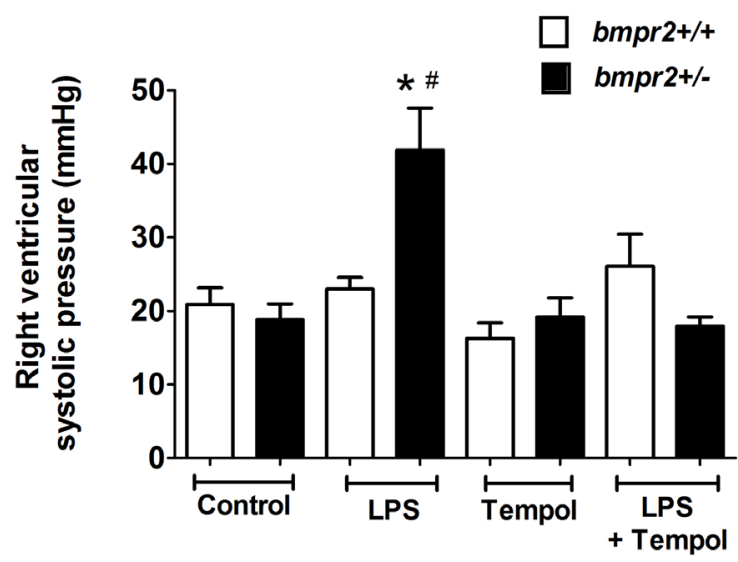

Fig.1: Bmpr2+/- mice exhibit increased right ventricular systolic pressures after chronic LPS administration. This is not seen in the wild-type, and is prevented by co-administration of Tempol, a superoxide dismutase mimetic.

Key:

${ }^{*} p<0.05$ compared to untreated bmpr2+/- mice $\# p<0.01$ compared to wild-type mice with LPS

Abstract T4 Figure 1

\section{T5 SAMPLING AIRWAY MUCOSAL LINING FLUID IDENTIFIES ROLES FOR IL-33 AND MULTIPLE INFLAMMATORY PATHWAYS IN VIRUS-INDUCED ASTHMA EXACERBATIONS}

doi:10.1136/thoraxjnl-2012-202678.005

'DJ Jackson, 'M Trujillo-Torralbo, 'J Footitt, ${ }^{2 B}$ Shamji, 'J del-Rosario, 'A Telcian, ${ }^{2}$ T Hunt, ${ }^{3} \mathrm{D}$ Hunt, ${ }^{1} \mathrm{P}$ Mallia, ${ }^{4} \mathrm{OM}$ Kon, ${ }^{2} \mathrm{M}$ Edwards, ${ }^{2} \mathrm{~J}$ Westwick, ${ }^{1} T$ Hansel, ${ }^{1} \mathrm{SL}$ Johnston. 'National Heart and Lung Institute, Imperial College London, MRC \& Asthma UK Centre in Allergic Mechanisms of Asthma, London, UK; ${ }^{2}$ Novartis Institute for Biomedical Research, UK; ${ }^{3}$ Hunt Developments Ltd, Midhurst, UK; ${ }^{4}$ mperial College NHS Trust

Rhinovirus (RV) infection is the most common cause of asthma exacerbations (AE), however mechanisms are poorly understood. Conventional sampling techniques such as bronchoalveolar lavage dilute many cytokines below limits of detection and consequently it has not been possible to measure key mediators of Th1, Th2 and Th17 pathways during virus-induced AE's. In addition, IL-33, an epithelial-derived alarmin, has recently been shown to be essential for mouse virus-induced airway hyperresponsiveness, however the relationship between IL-33 and exacerbations in human asthma remains unknown. Using the human model of experimental RV induced AE along with novel techniques to sample upper and lower airway mucosal lining fluid (MLF) we investigated the roles of IL-33 and several other prominent cytokines in virus-induced AE's.

Methods 32 mild-to-moderate asthmatics and 14 healthy subjects were inoculated nasally with RV-16. Symptom scores were recorded daily. Bronchoscopies were performed 2 weeks prior to inoculation and on $\mathrm{d} 4$ post-inoculation. Novel techniques to sample MLF called 'bronchosorption' and 'nasosorption' were performed. Cytokines were measured in both bronchial and nasal samples at baseline and on $\mathrm{d} 4$ with further nasal sampling on days 2, 3, 5, 7, 10 and 42.

Results In asthma, nasal IL-4, $-5,-13,-17,-33$, and IFN- $\gamma$ levels were significantly increased during infection compared to baseline (all P<0.001). IL-33 and Th2 cytokines but not IFN- $\gamma$ or IL-17 were increased in asthma compared to healthy subjects $(\mathrm{P}<0.01)$. In the asthmatic lung, relationships between bronchial IL-33 $(\mathrm{P}<0.05)$,
IL-13 $(p<0.05)$, IL-5 $(p=0.059)$ and chest symptom scores were observed. Bronchial levels of these cytokines correlated with nasal levels during infection $(\mathrm{p}<0.05)$ whilst baseline nasal levels of Th2 cytokines correlated strongly with infection levels $(p<0.001)$.

Conclusion Sampling MLF permits the direct measurement of previously undetectable mediators across multiple inflammatory pathways. Increased IL-33 and Th2 induction are associated with increased AE severity. IL-33 correlated strongly with Th2 cytokine levels and may represent a novel target for the treatment of virusinduced AE's. In addition, nasal Th2 inflammation correlated with bronchial levels whilst baseline levels predicted the magnitude of Th2 induction during the AE. Therefore it may be possible to use nasosorption to guide therapy with anti-IL-5 and anti-IL-13 mAb treatments.

\section{T6 THROMBOCYTOSIS IS ASSOCIATED WITH INCREASED SHORT AND LONG TERM MORTALITY AFTER EXACERBATION OF CHRONIC OBSTRUCTIVE PULMONARY DISEASE}

doi:10.1136/thoraxjnl-2012-202678.006

MT Harrison, P Short, P Williamson, A Singanayagam, AR Akram, JD Chalmers, S Schembri. NHS Tayside, Dundee, UK

Introduction There is increasing evidence suggesting that platelets play a significant role in inflammation in addition to their established role in thrombosis. Systemic inflammation is linked to poor short and long term outcomes in COPD Increased platelet activation has been reported in acute exacerbations of COPD (AECOPD). We investigated whether thrombocytosis is associated with poor outcomes following AECOPD.

Methods A prospective observational cohort study of patients hospitalised with AECOPD was performed. The dataset consists of patients $>40$ years, with spirometrically confirmed COPD admitted with exacerbations between 2009-2011. Platelet count was recorded on admission. The primary outcome was in-hospital mortality with secondary outcomes of 1 year all-cause mortality, cardiovascular events and mortality. Analyses were conducted using coxproportional hazards regression after adjustment for demographics, co-morbidities, chronic treatments and severity of exacerbations.

Results 1343 patients (49\% male) were included with a median age of 72 years (interquartile range 63-79 years). 157 patients $\left(11.7 \%\right.$ ) had thrombocytosis (platelet count $>400 \times 10^{9}$ cells $/ \mathrm{mm}^{3}$ ).

Thrombocytosis correlated with established markers of poor outcome in COPD, including hypoalbuminaemia (34 IOR 31-38 vs 37 33-40, p<0.0001), low BMI (BMI <18.5 in $14.6 \%$ vs $9.1 \%$, $\mathrm{p}=0.03$ ) and frequent exacerbations in the previous year (mean 1.42 standard deviation(SD) 1.9 vs mean 1.1 SD 1.8, $\mathrm{p}=0.02$ ).

Thrombocytosis was significantly associated with in-hospital mortality: Hazard ratio (HR) 1.96 (1.15-3.32) and 1 year mortality after discharge HR 1.48 (1.12-1.97). The relationships with cardiovascular hospitalisation HR 1.17 (0.84-1.64) and 1 year cardiovascular mortality HR $1.33(0.82-2.14)$ were not statistically significant.

To determine if the increased risk associated with thrombocytosis was potentially modifiable, we studied whether antiplatelet treatment (chronic prescription of aspirin or clopidogrel) would attenuate the risks associated with thrombocytosis. Anti-platelet treatment was associated with reduced 1 year mortality HR 0.75 (0.59-0.97) with a trend towards reduced in-hospital mortality HR 0.66 (0.40-1.09), cardiovascular hospitalisation HR 0.81 (0.61-1.08) and cardiovascular death HR 0.85 (0.57-1.26).

Conclusion Thrombocytosis was associated with increased in-hospital and 1 year mortality. This relationship was only partly attributable to increased cardiovascular mortality. Anti-platelet medications correlated with significantly lower 1 year mortality and may have a protective role to play in COPD. 